\title{
A Note on Construction of the Equivalent Plastic Strain Increment
}

\author{
C. A. Berg* \\ Institute for Basic Standards, National Bureau of Standards, Washington, D.C. 20234
}

(May 18, 1972)

\begin{abstract}
Strain hardening plastic deformation of a material possessing a yield locus which may be written as a homogeneous function of the stress components, and which obeys the classical associated flow rule for metals is considered. The material may be anisotropic and may display plastic dilatation. A method is given for constructing the equivalent plastic strain increment in such a way that the increment of plastic work is always equal to the product of the equivalent plastic strain increment and the equivalent yield stress. The method is implied in classical treatments of hardening but seems not to have been given explicitly heretofore.
\end{abstract}

Key words: Equivalent strain; hardening; plasticity; strain hardening; work hardening.

\section{Introduction}

From time to time one encounters the need to employ an equivalent plastic strain increment in plasticity. Most frequently this need arises in the construction of mathematical descriptions of strain hardening $[1,2] .^{1}$ Several different forms of equivalent plastic strain increment have been suggested, and often the physical basis for the suggestion is not made clear. The purpose of this note is to offer a physically based rule for the determination of the equivalent plastic strain increment. The rule is implied in classical treatments of the subject, but appears not to have been given explicitly.

\section{Plastic Deformation With Hardening}

We assume, as is conventional in plasticity theory, that the yield condition may be given in the form

$$
f\left(\sigma_{i j}\right)-Y(\text { history })=0,
$$

where $Y$ is the "equivalent yield stress" and depends upon the history of deformation so as to represent the hardening of the material. In this paper, $Y$ is numerically equal to the yield stress in uniaxial tension. The function $f\left(\sigma_{i j}\right)$ must, of course, have the physical dimensions of stress. We assume that $f\left(\sigma_{i j}\right)$ is a homogeneous function of degree unity in the stress components $\sigma_{i j}$. These assumptions imply that the yield locus merely expands without changing shape as the material hardens, with the magnitude of $Y$ determining the current size of the yield locus.

If one assumes (as in [3]) that hardening is determined by the plastic work $\left(\delta w^{p}\right)$ done on the material during each increment of plastic strain $\left(\delta \epsilon_{i j}^{p}\right)$, then one may construct an equivalent plastic strain increment to use in a theory of hardening as follows. The increment of plastic work $\left(\delta w^{p}\right)$ for each increment of plastic strain $\left(\delta \epsilon_{i j}^{p}\right)$ is

$$
\delta w^{p}=\sigma_{i j} \delta \epsilon_{i j}^{p},
$$

where the $\sigma_{i j}$ are the stress components which satisfy the yield condition and produce the required strain increment $\delta \epsilon_{i j}^{p}$. Now, the associated flow rule of plasticity theory requires that the plastic strain increment lie normal to the yield surface [4] (in the appropriate space) so that

$$
\delta \epsilon_{i j}^{p}=\delta \lambda\left(\frac{\partial f}{\partial \sigma_{i j}}\right)_{f-Y=0},
$$

where $\delta \lambda$ is a nonnegative scalar multiplier. In attempting to identify an equivalent plastic strain $\left(\delta \epsilon^{p}\right)$ one seeks a function of the plastic strain components $\left(\delta \epsilon_{i j}^{p}\right)$ with the property that the product of the equivalent plastic strain increment and the equivalent yield stress is always equal to the increment of plastic work.

$$
\delta \epsilon^{p} \cdot Y=\delta w^{p}=\sigma_{i j} \delta \epsilon_{i j}^{p}
$$

*Professor and Chairman, Department of Mechanical Engineering, The University of Pittsburgh. Presently Visiting Scientist, National Bureau of Standards, Washington, D.C.

${ }_{1}^{1}$ Figures in brackets indicate the literature references at the end of the paper. 
From eq (3)

$$
\delta w^{p}=\sigma_{i j} \delta \epsilon_{i j}^{p}=\delta \lambda \sigma_{i j} \frac{\partial f}{\partial \sigma_{i j}},
$$

and since $f\left(\sigma_{i j}\right)$ has been assumed to be a homogeneous function of degree one, Euler's theorem for homogeneous functions requires

$$
\sigma_{i j} \delta \epsilon_{i j}^{p}=\delta \lambda f=\delta \lambda Y,
$$

the last step coming from eq (1). The desired relationship

$$
Y \delta \epsilon^{p}=\delta w^{p}=\delta \lambda Y,
$$

is satisfied by setting

$$
\delta \epsilon^{p}=\delta \lambda .
$$

That is, the equivalent plastic strain increment is just the nonnegative scalar multiplier $\delta \lambda$ which provides the generalized length of the plastic strain increment eq (3).

If one uses the quantity

$$
|| \delta \epsilon||=\left(\delta \epsilon_{i j}^{p} \delta \epsilon_{i j}^{p}\right)^{1 / 2}=\left(\delta \epsilon_{1}^{2}+\delta \epsilon_{2}^{2}+\delta \epsilon_{3}^{2}\right)^{1 / 2}
$$

as a measure of the magnitude of the plastic strain increment, where $\delta \epsilon_{1}, \delta \epsilon_{2}$, and $\delta \epsilon_{3}$ are the principal value of plastic strain increment, then from eq (3)

$$
|| \delta \epsilon||=\delta \lambda\left(\frac{\partial f}{\partial \sigma_{i j}} \frac{\partial f}{\partial \sigma_{i j}}\right)^{1 / 2} .
$$

Thus the equivalent plastic strain increment can be given as

$$
\delta \lambda=\delta \epsilon^{p}=\frac{\sqrt{\delta \epsilon_{1}^{2}+\delta \epsilon_{2}^{2}+\delta \epsilon_{3}^{2}}}{\sqrt{\partial f / \partial \sigma_{i j} \partial f / \partial \sigma_{i j}}},
$$

for any yield locus $\left(f\left(\sigma_{i j}\right)\right)$ with which one happens to be concerned. For example, in the case of the von Mises yield locus, eq (l) becomes

$$
\left(3 / 2 s_{i j} s_{i j}\right)^{1 / 2}-Y=0,
$$

where $s_{i j}=\sigma_{i j}-1 / 3 \sigma_{k k} \delta_{i j}$, is the stress deviator. For this case, $\sqrt{\partial f / \partial \sigma_{i j} \partial f / \partial \sigma_{i j}}=\sqrt{3 / 2}$, and the equivalent plastic strain is given by

$$
\delta \epsilon^{p}=\sqrt{2 / 3 \delta \epsilon_{i j}^{p} \delta \epsilon_{i j}^{p}},
$$

which is the classical result. The use of eq (10) above as a rule for choosing the equivalent plastic strain increment may, at times, be complicated, because the quantity $\left(\partial f / \partial \sigma_{i j} \partial f / \partial \sigma_{i j}\right)$ must be evaluated point by point along the yield locus. However, despite such complications one hopes that the rule offered in eq (10) will be useful as a basis for constructing measures of equivalent plastic strain.

Once the equivalent plastic strain increment has been constructed it may be used as a variable to describe the history of deformation upon which hardening depends. With the net equivalent plastic strain $\epsilon^{p}$ given by

$$
\epsilon^{p}=\int \delta \epsilon^{p},
$$

(note: $\delta \epsilon^{p}>0$ so that $\epsilon^{p}$ increases monotonically in any deformation process), one may rewrite eq (1) as

$$
f\left(\sigma_{i j}\right)-Y\left(\epsilon^{p}\right)=0 .
$$

Equation (14) can now be tested by comparing experimental data from various deformation histories as in [1]. Furthermore, the rate of hardening determines the magnitude of plastic strain increment where the material is at yield, under the stresses $\sigma_{i j}$, and then is subjected to an increment of stress $d \sigma_{i j}$. One may rewrite eq (3) to give the components of plastic strain increment $\delta \epsilon_{i j}^{p}$ in terms of the hardening rate $Y^{\prime}$ and the increase in yield stress $d Y$ required by the stress increment $d \sigma_{i j}$;

$$
\delta \epsilon_{i j}^{p}=\frac{d Y}{Y^{\prime}} \frac{\partial f}{\partial \sigma_{i j}} .
$$

The convenient formula (15) is the classical result of plasticity theory, but its derivation in the present note appears somewhat simpler than those usually found in the literature.

\section{References}

[1] Sidebottom, O. M. (1971), Note on effective plastic strain for a Tresca material, J. Appl Mech (Dec. 1971).

[2] Sato, Y., and Shegunice, S. (1971), Yielding of mild steel under biaxial state of uniform stress, Bul Japan Soc M.E. 14, 72.

[3] Hill, R. (1950), The Mathematical Theory of Plasticity (Clarendon Press, Oxford, England).

[4] Bishop, J., and Hill, R. (1951), A theory of the "Plastic distortion of a polycrystalline aggregate under combined stress," Phil Mag 42, 414-427.

(Paper 76Cl\&2-332) 